\title{
Processing of written and spoken words: Evidence for common coding
}

\author{
VICKI L. HANSON \\ Salk Institute for Biological Studies, La Jolla, California 92138
}

\begin{abstract}
Common processing systems involved during reading and listening were investigated. Semantic, phonological, and physical systems were examined using an experimental procedure that involved simultaneous presentation of two words: one visual and one auditory. Subjects were instructed to attend to only one modality and to make responses on the basis of words presented in that modality. Influence of unattended words on semantic and phonological decisions indicated that these processing systems are common to the two modalities. Decisions in the physical task were based on modality-specific codes operating prior to the convergence of information from the two modalities.
\end{abstract}

Studies concerned with word processing have tended to approach the topic by investigating either reading or listening, exclusively, largely ignoring the question of common processing systems for the two tasks. Yet, at some level of stimulus analysis, printed words and spoken words must share processing. The present research examines the relationship between the internal codes involved during reading and during listening and investigates at what level ${ }^{1}$ of stimulus analysis it might be possible to merge the research from these two areas.

Evidence from bisensory tasks bears upon the question of common semantic coding of visually and auditorily presented words. Lewis (1972) and Sen and Posner (1979) have found facilitation in pronunciation latency for attended words in either modality when the same word is simultaneously presented on the unattended modality. Similarly, when an unattended auditory digit is the same as the attended visual digit, pronunciation latency for the digit is facilitated (Greenwald, 1970; Mynatt, 1977). It has also been shown that unattended visual (Lewis, 1972) and auditory (Greenwald, 1970; Mynatt, 1977) words and digits interfere with pronunciation of items presented to the attended modality when the attended and unattended items are semantically related. This influence of semantically related unattended words indicates automatic activation of a semantic code that is shared by the two modalities.

This paper is based on a dissertation submitted to the graduate school of the University of Oregon in partial fulfillment of degree requirements. I am grateful to Michael Posner for his guidance throughout this project. I also wish to thank Ursula Bellugi and Eleanor Gibson for their helpful comments on earlier drafts of the manuscript and Fred Chang for his assistance with data analysis. The investigation and writing of this report were supported by National Institute of General Medical Sciences Training Grant 5 T01 GM 02165 BHS and by National Institutes of Health National Research Service Award 1 F32 NS06109-01 from the Division of Neurosciences and Communicative Disorders and Stroke. Requests for reprints should be sent to Haskins Laboratories, 270 Crown Street, New Haven, CT 06510.
During reading and listening tasks, is there similarly automatic activation of a shared phonological code? For the present purpose, this does not encompass the question of whether a phonological code involved during reading might be accessed prior or subsequent to lexical access (see Coltheart, 1978). Of interest, rather, is whether written and spoken inputs share processing at the level of phonological analysis.

The present study examines common processing of written and spoken words for three different coding systems: semantic, phonological, and physical. While, by definition, the physical codes are modality specific, thus not making the concept of a common physical code reasonable, it is possible that no decisions about words can be made prior to identification at some common lexical or semantic level. The necessity of first accessing a common code is addressed here.

The procedure of the present experiment involved presentation of the same stimuli for all three systems, but task instructions varied the level of stimulus analysis needed for response decisions. In each case, the subjects' task required a two-choice response. On each trial, there was simultaneous presentation of two words: one visual and one auditory. Subjects were instructed to attend to one modality and asked to make responses on the basis of the word presented in that modality. Of concern was the influence of words presented in the unattended modality. If responses were based on a common code, then unattended words should influence decisions about attended words.

For the semantic task, subjects were asked to determine whether or not the attended word belonged to a specific semantic category. In line with previous studies, it was expected that there would be facilitation for redundant trials (i.e., on which the same word is presented to both modalities) compared with control trials on which only the attended word belonged to the target category.

Redundancy gains could result from the redundant name information and/or from the redundant category 
information. It is possible to separate the contributions of these two sources to any redundancy gains by including a condition in which both the attended and unattended words on a trial are different instances of the same category. If the category of the unattended word is automatically activated, there should be facilitation in that condition relative to a control condition in which only the attended word belonged to the target category. Facilitation for redundant trials in excess of the facilitation produced for these same-category/differentnature trials can be attributed to redundant name information.

Interference may be expected in a condition in which only the word in the unattended modality belongs to the target category. Interference would result from the two words' activating semantic codes that lead to a response conflict, with the unattended word leading to an incorrect answer. The control for the test of interference is a condition in which neither the attended nor the unattended items on the trial belong to the relevant category.

The phonological task was a phoneme-monitoring task, in which subjects were asked to decide whether or not a word contained a target phoneme. The phonememonitoring task was here extended to monitoring written words for the sounds they represent. Of interest was whether words in the unattended modality influenced phoneme-monitoring decisions about attended words. Facilitation would be expected for redundant trials due either to name or to phoneme redundancy. If phoneme redundancy contributes to redundancy gains, there should be facilitation on trials in which the attended and unattended words, although different, both contain a target phoneme. The control condition for the tests of facilitation was trials on which a target phoneme was present only in one modality. Interference effects were measured by comparing a condition in which only the unattended word contained a target with a condition in which neither the attended nor the unattended word contained a target phoneme. Interference would be produced if the results of phonological analysis of the two signals led to conflicting response decisions.

For the physical task, subjects were asked to make decisions about nonlinguistic properties of the stimuli presented. If the ability to make even these nonlinguistic decisions about words is dependent upon the activation of a code common to the two modalities, there should be redundancy gains when the same word is presented in both the attended and unattended modalities. If, on the other hand, physical decisions can be made on the basis of the modality-specific codes operating prior to convergence of information from the two modalities, then no redundancy gains would be produced.

\section{METHOD}

\section{Stimulus Materials}

Stimuli were 72 words taken from the Battig and Montague (1969) category norms. There were 12 words from each of the following six commonly produced categories (Herrmann \&
Table 1

Trial Types in the Semantic Task

\begin{tabular}{|c|c|c|c|c|}
\hline \multirow[b]{2}{*}{ Trial Type } & \multicolumn{2}{|c|}{ Modality } & \multirow[b]{2}{*}{ Member? } & \multirow[b]{2}{*}{ Trials } \\
\hline & Attended & Unattended & & \\
\hline REDUNDANT & chair & chair & Yes & 4 \\
\hline BOTH & chair & lamp & Yes & 4 \\
\hline ATTENDED & chair & mouth & Yes & 4 \\
\hline UNATTENDED & mouth & chair & No & 6 \\
\hline NEITHER & mouth & beets & No & 6 \\
\hline
\end{tabular}

Note-The items given are for a situation in which the target category is FURNITURE. "Member?" indicates whether the attended word is a member of the target category. "Trials" indicates the number of trials per block.

Kay, 1977): furniture, relatives, parts of the body, clothing, animals, and vegetables. In each category, words were selected such that half the words contained the target phonemes $/ \mathrm{s} /$ and $/ z /$ and half did not. To discourage a strategy of visual search for the grapheme " $S$ " in the phonological task, the stimulus list included the words "niece," "celery," "lettuce," and "fox," in which a target phoneme was present but spelled with the letters " $C$ " or " $X$ " rather than "S." To further discourage such a strategy, the words "shirt," "shoe," and "sheep," which contained the letter "S" but not a target phoneme, were also included in the list. An attempt was made to equate the frequency of production for the target and nontarget words according to the Battig and Montague norms. The stimulus words used are listed in the Appendix.

Two 45-min stimulus tapes were prepared: one for the visual-attend condition and one for the auditory-attend condition. Six sets of trials were recorded on each audiotape at a mean intertrial interval of $2 \mathrm{sec}$ by a male and a female speaker. Each set contained six blocks of 24 trials, and the blocks in each set were all related to the same category, as defined by the semantic task. Stimulus tapes were prepared such that the unattended stimuli in one condition were the attended stimuli in the other condition. Each of the 72 stimulus items occurred an equal number of times in the attended and unattended modalities.

On each trial, there was simultaneous presentation of a visual and an auditory word. The relationship between these two words varied, creating different semantic, phonological, and physical trial types.

Semantic trial types. In the semantic task, subjects were asked to decide whether or not the 24 attended items of a block belonged to a specific category. The semantic trial types were defined in terms of whether items in the attended and/or unattended modality belonged to this category. The five trial types are shown in Table 1. Shown in the table is a situation in which the target category is "furniture." For $50 \%$ of the trials, the items in the attended modality belonged to the target category.

Phonological trial types. Targets in the phonological task were the phonemes $/ \mathrm{s} /$ and $/ \mathrm{z} /$. The six phonological trial types varied as to whether a target phoneme was present in the attended and/or the unattended modality. Trials were arranged so that a target sound was present on the attended modality on $50 \%$ of the trials and was absent on the attended modality on $50 \%$ of the trials. The six trial types are shown in Table 2.

Physical trial types. Physically, the visual stimuli varied in that the words could be printed in either capital or lowercase (small) letters. The auditory stimuli were spoken in either a male or female voice. There were thus four stimulus combinations: capital-male, small-male, capital-female, and small-female. Each combination occurred on $25 \%$ of the trials. For $1 / 6$ of the words in each combination, the same word was presented in the attended and unattended modalities. These were the REDUNDANT trial types. Trials on which different words were presented in the two modalities were the NONREDUNDANT trial types. 
Table 2

Trial Types in the Phonological Task

\begin{tabular}{lcccc}
\hline \multicolumn{4}{c}{ Trial Type } & \multicolumn{2}{c}{ Modality } & & \\
\cline { 2 - 4 } Attended & Unattended & Sound? & Trials \\
\hline REDUNDANT-YES & skunk & skunk & Yes & 2 \\
BOTH & skunk & niece & Yes & 4 \\
ATTENDED & skunk & wolf & Yes & 6 \\
UNATTENDED & wolf & son & No & 6 \\
NEITHER & wolf & shirt & No & 4 \\
REDUNDANT-NO & wolf & wolf & No & 2 \\
\hline
\end{tabular}

Note - "Sound?" indicates whether target sound was present in the attended modality. "Trials" indicates the number of trials per block.

\section{Design}

There were three tasks: semantic, phonological, and physical. Within each task, the semantic, phonological, and physical relationships between the two words presented on a trial were varied. Subjects performed all three tasks in separate sessions under either visual-attend instructions or auditory-attend instructions. Attended modality was thus the only between-subjects factor.

\section{Procedure}

The order of task presentation was counterbalanced across subjects in the two attend conditions. Each subject was tested three times with the same stimulus tape, once in each of the three sessions. Tapes were played at a comfortable listening intensity on a Revox Model A77 tape recorder located in an adjacent room. Subjects listened to the experimental tapes through a set of headphones.

Visual and auditory word presentations were synchronized so that the onsets of the two words were simultaneous. Reaction time (RT) was measured from stimulus onset. Synchronization was accomplished using a device that detected the onset of the recorded spoken words. This detection triggered the immediate display of the written words on an oscilloscope. A response from the subject terminated the visual word display. If the subject had not responded within $1 \mathrm{sec}$ after stimulus onset, the display was terminated and there was no response recorded for that trial. Visual presentations and data collection were controlled by a PDP-15 computer.

Subjects were informed that after every 24 trials, they would get feedback as to the number of correct trials in the block and their mean RT for that block. The feedback was visually displayed for $5 \mathrm{sec}$. There was a brief rest period following each set.

In each of the three tasks, subjects were asked to make a two-choice response, and decisions were indicated by pressing one of two response keys. Subjects were instructed that a visually and an auditorily presented word would be simultaneously presented on each trial, and they were told that only the words on one modality would be relevant to the task. The instructions in the auditory-attend condition emphasized that subjects were to keep looking at the visually presented words. These subjects were questioned at the end of each session as to whether they, in fact, did look at the written words. All subjects indicated that they had followed the instructions.

Semantic task. In the semantic task, a category name was visually displayed for $2 \mathrm{sec}$ before the start of each block. This presentation indicated the target category for the following block of trials. Subjects were instructed to decide whether the attended word on each trial belonged to that (target) category. A specific category was the target category for six consecutive blocks (i.e., one set).

Phonological task. In the phonological task, subjects were instructed to decide whether or not the attended word contained an $/ s /$ or $/ z /$ sound. They were told that this did not necessarily mean that the word was spelled with the letter "S." Examples of words containing a target (e.g., "jacks," "ice," "ax," and "rose") were given.

Physical task. On visual-attend trials, subjects were asked to decide whether a word was presented in capital or small letters. For trials in the auditory-attend condition, subjects were asked to decide whether the word they heard was spoken in a male or a fermale voice.

\section{Subjects}

Subjects were 12 paid volunteers from the subject pool at the Cognitive Laboratory at the University of Oregon. All were native English speakers and had normal hearing and normal or corrected-to-normal vision. Subjects were paid $\$ 2.50 / \mathrm{h}$ and were tested in three separate sessions on consecutive days. Six subjects performed the tasks under visual-attend instructions, and six performed the tasks under auditory-attend instructions.

\section{RESULTS AND DISCUSSION}

Analyses were performed on median correct RTs and on mean percentage errors. ${ }^{2}$ The criterion for significance was set at a probability level of .05 . Results will be presented and discussed for each of the three tasks separately.

One general comment is in order, however, before proceeding with the separate discussions. RTs and errors were comparable across the auditory-attend and visualattend conditions of the three tasks. Overall, there were no significant RT or error differences across the two conditions of the tasks.

\section{Semantic Task}

Results for "yes" and "no" responses were analyzed separately to test for facilitation and interference effects, respectively. The results of the semantic task are given in Table 3.

Analysis of trial type by attended modality for "yes" response RTs resulted in a main effect of trial type both for the analysis of subjects $[F(2,20)=23.24$, $\mathrm{MSe}=168.05]$ and the analysis of items $[\mathrm{F}(2,284)=$ $42.55, \mathrm{MSe}=1,046.88]$. The effect was also significant when both were simultaneously considered $\left[\mathrm{min} \mathrm{F}^{\prime}(2,46)\right.$ $=15.03]$. Newman-Keuls analyses revealed that there was facilitation for REDUNDANT and BOTH trials relative

Table 3

Results of the Semantic Task

\begin{tabular}{lcccc}
\hline & \multicolumn{2}{c}{ Auditory-Attend } & \multicolumn{2}{c}{ Visual-Attend } \\
\cline { 5 - 6 } \cline { 5 - 6 } & Mean & PE & Mean & PE \\
\hline & \multicolumn{4}{c}{ "Yes" Responses } \\
REDUNDANT & 503 & 5.0 & 471 & 5.7 \\
BOTH & 528 & 6.9 & 483 & 7.1 \\
ATTENDED & 553 & 8.1 & 493 & 9.0 \\
& & “No" & Responses & \\
UNATTENDED & 572 & 8.7 & 525 & 8.8 \\
NEITHER & 568 & 6.6 & 519 & 5.2 \\
\hline
\end{tabular}

Note - Mean $=$ mean of the median correct RTs (in milliseconds); $P E=$ mean percentage errors. 
to ATTENDED trial types. Additionally, REDUNDANT trials were faster than BOTH trial types. An interaction of Trial Type by Attended Modality was indicated both in the subjects analysis $[\mathrm{F}(2,20)=3.56, \mathrm{MSe}=168.05]$ and in the items analysis $[\mathrm{F}(2,284)=5.49$, $\mathrm{MSe}=$ $1,046.88]$, but it failed to reach significance in the simultaneous consideration of subjects and items $\left[\min F^{\prime}(2,53)=2.16, n . s.\right]$. There is thus some question as to the reliability of the interaction. Separate analyses showed that, in fact, there was significant facilitation produced in each condition alone [auditory-attend, $\mathrm{F}(2,10)=20.39$, $\mathrm{MSe}=185.19$; visual-attend, $\mathrm{F}(2,10)=$ $4.84, \mathrm{MSe}=150.52]$.

With errors, there was a main effect of semantic trial type for subjects $[\mathrm{F}(2,20)=103.04, \mathrm{MSe}=.31]$ and items $[F(2,284)=23.37$, MSe $=30.67]$, as well as for the quasi $F\left[\min F^{\prime}(2,247)=19.05\right]$. In agreement with the RT data, Newman-Keuls analyses indicated that there were significantly fewer errors on REDUNDANT and BOTH trial types than on ATTENDED trials, and also fewer errors for REDUNDANT trials than for BOTH trials. There was no interaction between attended modality and semantic trial type, as indicated by the subjects analysis, which did not reach significance $[F(2,20)=$ $1.44, \mathrm{MSe}=.31]$.

Prior research (Greenwald, 1970; Lewis, 1972; Mynatt, 1977) has shown that semantically related material in an unattended modality, such as the situation here for BOTH trial types, produced interference in task performance. But it must be remembered that in the previous studies the response was to pronounce the attended stimulus. Therefore, semantically related stimuli in the attended and unattended modalities led to different and competing responses. In the present study, the two words presented on BOTH trials were semantically related in that they both came from the same semantic category and thus, for the purposes of the semantic task used here, led to the same response decision.

Facilitation is the result of pathway activation. When a code is shared by two stimuli presented in close temporal proximity, the processing of the second of the two stimuli is facilitated. That is, the internal representation of the word remains active for some brief period of time following stimulus presentation. When an item that shares the same internal coding is presented during this period, processing of the second item is facilitated. Kirsner and Smith (1974) have shown that this facilitation occurs for a word repeated within a modality, as well as for cross-modality repetitions of a word.

The response facilitation for REDUNDANT and BOTH trials indicates that the unattended words activate a coding pathway that is shared by the attended and unattended words. The facilitation for REDUNDANT trial types is in accord with previous studies that have indicated that there are redundancy gains when the same word is presented to the attended and unattended modalities (Lewis, 1972; Sen \& Posner, 1979). Inclusion of BOTH trial types here allows for an analysis of different sources contributing to these redundancy gains. For REDUNDANT trials, the attended and unattended words share both name and semantic category identity. For BOTH trial types, the two words share only semantic category identity. The greater facilitation for REDUNDANT trials than for BOTH trials indicates that there is more of a shared activation pattern for the written and spoken words on REDUNDANT trials than for the two words presented on BOTH trials. That is, name as well as category redundancy contribute to the facilitation for REDUNDANT trials. There is thus a shared name code for written and spoken words in addition to the shared semantic code.

It should be noted that facilitation produced by the unattended words did not disappear with practice. Trials were divided into those belonging to the first and second halves of the test blocks. An analysis indicated no interaction of practice by trial type. There was, therefore, the same pattern of results in both the first and second halves of the test blocks.

Analysis of errors for "no" responses found that interference for UNATTENDED trial types relative to NEITHER trial types was not reliably produced. An effect obtained from the subjects analysis $[F(1,10)=$ $234.54, \mathrm{MSe}=.21]$ was not supported by the analysis of items $[F(1,142)=3.54$, $\mathrm{MSe}=33.90$, n.s. $]$, nor by the analysis considering subjects and items simultaneously $\left[\min F^{\prime}(1,146)=3.49\right.$, n.s.] . An interaction of Attended Modality by Semantic Trial Type for subjects $[F(1,10)=17.36, \mathrm{MSe}=.21]$ similarly did not reach significance for the items analysis $[F(1,142)=$ $\left.4.30, \mathrm{MSe}=33.90 ; \min \mathrm{F}^{\prime}(1,118)=3.45\right]$.

The lack of reliable interference indicates that unattended words did not tend to influence responding at the decision level. Facilitation for REDUNDANT and BOTH trial types could result from activation of common pathways and/or from response compatibility of the attended and unattended words. In addition, the lack of interference suggests that the facilitation for BOTH trials was caused by activation of common pathways for attended and unattended words.

In summary, results of the semantic task indicate that there is automatic activation of the name and semantic category for both written and spoken words. In addition, the name and semantic information are represented in coding systems common to the two modalities. This common coding was evidenced in the facilitation for BOTH trial types, the result of shared semantic codes for the written and spoken words, and in the greater facilitation for REDUNDANT than for BOTH trial types, the result of shared code names.

\section{Phonological Task}

Results of the phonological task are given in Table 4. Preliminary inspection indicated very different patterns of results in the auditory-attend and visual-attend 
Table 4

Results of the Phonological Task

\begin{tabular}{|c|c|c|c|c|c|c|c|c|}
\hline & \multicolumn{2}{|c|}{ Auditory-Attend } & \multicolumn{2}{|c|}{ Visual-Attend } & \multicolumn{2}{|c|}{$\begin{array}{c}\text { Visual-Attend: } \\
\text { Delay } \\
\end{array}$} & \multicolumn{2}{|c|}{$\begin{array}{l}\text { Visual-Attend: } \\
\text { Delay CX Trials }\end{array}$} \\
\hline & Mean & $\mathrm{PE}$ & Mean & $\mathrm{PE}$ & Mean & $\mathrm{PE}$ & Mean & $\mathrm{PE}$ \\
\hline & \multicolumn{8}{|c|}{ "Yes" Responses } \\
\hline $\begin{array}{l}\text { REDUNDANT-YES } \\
\text { BOTH } \\
\text { ATTENDED }\end{array}$ & $\begin{array}{l}533 \\
586 \\
603\end{array}$ & $\begin{array}{l}9.2 \\
8.3 \\
9.1\end{array}$ & $\begin{array}{l}464 \\
477 \\
466\end{array}$ & $\begin{array}{r}10.9 \\
6.0 \\
5.9\end{array}$ & $\begin{array}{l}519 \\
538 \\
520\end{array}$ & $\begin{array}{l}8.5 \\
8.2 \\
6.1\end{array}$ & $\begin{array}{l}542 \\
569 \\
616\end{array}$ & $\begin{array}{l}37.8 \\
23.3 \\
33.7\end{array}$ \\
\hline ATTENDED & \multicolumn{8}{|c|}{ "No" Responses } \\
\hline $\begin{array}{l}\text { UNATTENDED } \\
\text { NEITHER } \\
\text { REDUNDANT-NO }\end{array}$ & $\begin{array}{l}629 \\
607 \\
573\end{array}$ & $\begin{array}{l}14.3 \\
12.6 \\
11.9\end{array}$ & $\begin{array}{l}516 \\
521 \\
506\end{array}$ & $\begin{array}{l}14.4 \\
14.5 \\
13.4\end{array}$ & $\begin{array}{l}535 \\
521 \\
508\end{array}$ & $\begin{array}{l}8.9 \\
9.3 \\
6.4\end{array}$ & 545 & 11.5 \\
\hline
\end{tabular}

Note-Mean = mean of the median RTs for correct responses (in milliseconds); $P E=$ mean percentage errors.

conditions. The two conditions were therefore analyzed separately.

Auditory attend. As in the semantic task, facilitation was produced for "yes" responses when the visually and auditorily presented words shared processing. There was a main effect of RT for phonological trial type "yes" responses $[F(2,10)=13.13, \mathrm{MSe}=597.39]$. The effect was supported by the items analysis $[F(2,119)=$ $9.55, \mathrm{MSe}=5,512.19]$ and the simultaneous consideration of both subjects and items $\left[\min F^{\prime}(2,49)=5.53\right.$ ] As in the semantic task, the facilitation was not eliminated by practice. Post hoc Newman-Keuls comparisons indicated that these results reflected redundancy gains for REDUNDANT-YES trials compared with BOTH and ATTENDED trial types. This absence of significant facilitation for BOTH trials indicates that activation of pathways for individual phonemes was not present. The analysis of errors did not reach significance for "yes" responses $[\mathrm{F}(2,10)=2 \cdot 10, \mathrm{MSe}=.73]$. Redundancy gains were not evident for REDUNDANT-NO trials compared with NEITHER trials [for RTs, $F(1,5)=3.59$, MSe $=965.00$, n.s.; for errors, $F(1,5)=2.64$, MSe $=.61$, n.s.] .

Interference for UNATTENDED trial types relative to NEITHER trial types was indicated in the error data for the subjects analysis only $[F(1,5)=69.92$, MSe $=$ .12]. The effect did not reach significance for the items analysis $\left[\mathrm{F}(1,63)=2.40, \mathrm{MSe}=659.12 ; \mathrm{min}^{\prime}\right.$ $(1,67)=2.32]$. RTs also showed no significant interference $[F(1,5)=4.96, \mathrm{MSe}=301.73]$.

The RT facilitation for "yes" response trials indicated that this task, which required a phonemic analysis of speech, was influenced by the unattended written words. This influence suggests that visually presented words are being processed by the same phonological system used for analyzing speech inputs. In related work, Rollins and Hendricks (1980) similarly concluded that written and spoken words are analyzed in a shared phonological system. They showed that in a divided attention task, subjects were poor at making rhyming decisions about visually presented words when attending to a simultaneous auditory input. The present work showed that activation of this common code occurred even in the absence of attention being directed to the processing of a word.

Visual attend. Analyses of RTs and errors in the visual-attend condition found no evidence of either facilitation or interference.

Testing for facilitation of "yes" responses, the effect of trial type on RTs $[F(2,10)=4.12$, MSe $=66.72]$ was not supported by the items analysis $[F(2,118)=$ $.12, \mathrm{MSe}=1,729.58 ; \min \mathrm{F}^{\prime}(2,128)=.12$, n.s.]. For errors, an effect of phonological trial type in the subjects analysis $[\mathrm{F}(2,10)=34.40, \mathrm{MSe}=142.05]$ was also not supported in the items analysis $[\mathrm{F}(2,121)=1.96, \mathrm{MSe}=$ 130.92 ; $\min F^{\prime}(2,130)=1.85$, n.s.] . Analysis of "no" response trials found no significant $\mathrm{RT}$ facilitation for REDUNDANT $-N O$ trials $[F(1,5)=6.48, \mathrm{MSe}=95.13]$. The analysis of errors was significant for subjects $[F(1,5)$ $=7.21, \mathrm{MSe}=.52]$, but this effect was not supported in the items analysis $[\mathrm{F}(1,58)=.06, \mathrm{MSe}=371.45$; $\min F^{\prime}(1,59)=.06$, n.s.] .

Interference for UNATTENDED trial types was not indicated for either RTs $[F(1,5)=.33, \mathrm{MSe}=181.15]$ or errors $[F(1,5)=.01, \mathrm{MSe}=2.16]$.

These results appear to indicate that phonemes of unattended spoken words do not influence visual phoneme monitoring. It seems a surprising finding that written words influence decisions about phonological properties of spoken words but spoken words do not influence decisions about phonological properties of written words. Two possible reasons for this asymmetric effect are immediately apparent: (1) Temporal differences in the presentation of written and spoken words mask the influence, and (2) subjects in the visualattend condition were doing a visual search task rather than monitoring for phonemes. These two possibilities were examined.

Visual attend: Delay. There are differences in temporal display characteristics for written and spoken words. For spoken word presentations, the stimulus must be displayed over a period of time. In the visualattend condition, therefore, phoneme decisions may be made before the information in the unattended words has a chance to influence responding. To test this 
possibility, the visual-attend condition of the phonological task was run again, using a delay in visual word presentation.

Because RTs in the phonological task had been approximately $100 \mathrm{msec}$ faster in the visual-attend condition than in the auditory-attend condition, the onset of the visual word was delayed by $100 \mathrm{msec}$ relative to the onset of spoken words in the new condition. RT was measured from the onset of the visually presented word.

Six subjects, none of whom had previously participated in the experiment, were recruited from the same subject pool as the earlier subjects. They were tested for one session in the visual-attend condition of the phonological task. All had the same qualifications as the earlier subjects.

Results of the delay condition are given in Table 4. As in the simultaneous condition, there was no reliable facilitation or interference. To understand this lack of effect, an analysis was made to determine whether subjects consistently monitored the visually presented words for phonemes or whether, in fact, subjects had a tendency to respond on the basis of the graphemic representation of the words. Subjects in the visualattend condition may have been performing a visual letter search rather than monitoring for the target phonemes. That is, they may have been searching for the grapheme "S" rather than the sounds represented by the spelled words. The instructions explained that they were not to just look for an "S," but it is still possible that some subjects adopted this strategy. Therefore, an analysis was performed on only those trials on which the target phonemes were represented orthographically by the letters " $\mathrm{C}$ " or "X," as in the words "niece" and "fox." For "yes" responses, these were trials on which the attended word contained a target phoneme spelled with the letter "C" or "X." For "no" responses, a target phoneme on the unattended modality was spelled with one of these letters. Given in Table 4 are the mean RTs and mean percentage errors for these CX trials of the visual delay condition.

Examining the four trial types that could contain CX words, there was evidence that, indeed, subjects monitored for the grapheme " $\mathrm{S}$ " in addition to monitoring for the target phonemes. As would be expected if subjects were searching for a printed "S," subjects analyses of "yes" responses found that there were more errors $[F(1,5)=9.98, \mathrm{MSe}=519.40]$ and longer RTs $[\mathrm{F}(1,5)=16.09, \mathrm{MSe}=1,398.67]$ for $\mathrm{CX}$ trials of the delay condition than for all trials of the delay condition combined. Additionally, there was an interaction of Trial Type by Target Sound $[\mathrm{F}(2,20)=6.41, \mathrm{MSe}=754.45]$, indicating different RT patterns for CX trials and for all trials combined.

For correct CX trials, subjects could not have based their responses on a search for the grapheme "S." While there are relatively few CX words, and therefore conclusions from these trials alone must be viewed with caution, it is still interesting to examine the influence of the unattended spoken words on these trials.

Examining CX trials for only the "yes" responses, RTs showed redundancy gains both in the subjects analysis $[F(2,10)=4.10, \mathrm{MSe}=2,069.32]$ and in the items analysis $[F(2,6)=18.17, \mathrm{MSe}=632.19]$. When both were simultaneously considered, however, the effect failed to reach significance $\left[\min F^{\prime}(2,14)=3.35\right.$, $\mathrm{p}<.10]$, causing some concern as to the simultaneous generality of the facilitation effect. However, this facilitation was similar to the redundancy gains produced in the auditory-attend condition of the phonological task. Also similar to that condition, there was no significant effect of errors for "yes" response trials $[\mathrm{F}(2,10)=1.66, \mathrm{MSe}=199.65]$.

Two factors thus have been identified that contributed to the asymmetric auditory-attend and visualattend results of the task: (1) the fact that spoken words must be displayed over time and (2) a tendency to search for the grapheme " $S$ " in the visual-attend condition. These two factors make the results of the visualattend condition, by themselves, difficult to interpret. However, the results of the auditory-attend condition were clear, and the similar results from the delay CX condition do lend support to conclusions based on the results of the auditory-attend condition.

The phoneme-monitoring task of the present experiment was used to determine if there is a phonological processing system common to the two modalities. As such, influence of the unattended words was examined. Results showed that unattended words facilitated phonological decisions about words presented on the attended modality, indicating automatic phonological analysis of written and spoken words in a code common to the two modalities.

\section{Physical Task}

REDUNDANT and NONREDUNDANT trials were compared in the physical task. Mean RTs and percentage errors for the two trial types in the auditory-attend and visual-attend conditions are shown in Table 5. Results of correlated $t$ tests indicated no redundancy gains for either RTs [visual attend, $\mathrm{F}(1,5)=.02, \mathrm{MSe}=1,344.44$; auditory attend, $\mathrm{F}(1,5)=.06, \mathrm{MSe}=10,978.98$, n.s.] or errors [visual attend, $\mathrm{F}(1,5)=.49, \mathrm{MSe}=6.74$; auditory attend, $\mathrm{F}(1,5)=.33, \mathrm{MSe}=.24$, n.s.]. It appears, therefore, that redundant information does not facilitate responding at the physical level.

Table 5

\begin{tabular}{|c|c|c|c|c|}
\hline \multirow[b]{2}{*}{ Trial Type } & \multicolumn{2}{|c|}{ Auditory-Attend } & \multicolumn{2}{|c|}{ Visual-Attend } \\
\hline & Mean & PE & Mean & $\mathrm{PE}$ \\
\hline REDUNDANT & 335 & 6.9 & 399 & 5.9 \\
\hline NONREDUNDANT & 350 & 6.7 & 401 & 4.9 \\
\hline
\end{tabular}


The fact that there was no influence of the unattended words in the physical task is important for two reasons. First, these results demonstrate that redundancy in the two modalities does not always facilitate responding to the attended words. Rather, the influence is confined to certain types of tasks. This selective facilitation lends importance to the influence of the unattended words in the semantic and phonological tasks, indicating that the facilitation produced in those tasks can be clearly attributed to common semantic and phonological coding.

Second, the results of the physical task indicate that decisions in the task were based on modality-specific visual and auditory codes operating prior to convergence of the information from the two modalities. Had a common name code been activated, there would have been redundancy gains. While studies have indicated that decisions about parts of words may at times be more difficult than decisions about whole words for both written (Reicher, 1969; Wheeler, 1970) and spoken (McNeill \& Lindig, 1973) inputs, the physical task used here clearly indicates that there are situations in which there can be some analysis of words prior to this convergence.

The fact that decisions about physical properties of words can be made without first taking higher order information into account does not mean, however, that these higher levels of information cannot influence decisions based on a physical code. Indeed, Rosch (1975) has shown that matching of physically identical items is affected by a category prime. The implication of the present finding for speaker and case judgments is that there are some properties of written and spoken words that are represented in a code specific to the input modality and that it is possible to make decisions on the basis of these modality-specific codes.

\section{GENERAL DISCUSSION}

The same stimuli were presented to subjects under three levels of stimulus analysis and under either visual attend or auditory-attend instructions. Results showed that words presented in the unattended modality that were related to words in the attended modality facilitated responding in the semantic and phonological tasks, but had no facilitatory effect in the physical task. Written and spoken words, it is therefore indicated, share semantic and phonological processing but have separate modality-specific codes that operate on information prior to the convergence of information from visual and auditory inputs.

This influence of unattended words on semantic and phonological decisions also indicates that the activation of semantic and phonological codes is automatic for both written and spoken word presentations. The activation is automatic in the sense that attention need not be directed at an input. The evidence for activation of a code related to a phonemic analysis of the visual words is indeed intriguing. While the current work does not indicate what use readers make of the phonemic analysis, it does indicate that a speech-related code is activated during the processing of written words.

The present analysis offers a basis for determining the circumstances under which written and spoken language are similarly processed. This common coding predicts that reading and listening studies should find similar results when semantic and phonological aspects of the tasks are investigated (Posner \& Hanson, 1980). The separate modality-specific visual and auditory codes, however, indicate that there are some aspects of reading and listening that are unique to the input modality.

\section{REFERENCES}

Battig, W. F., \& Montague, W. E. Category norms for verbal items in 56 categories: A replication and extension of the Connecticut category norms. Journal of Experimental Psychology Monograph, 1969, 80(No. 3, Pt. 2).

Coltheart, M. Lexical access in simple reading tasks. In G. Underwood (Ed.), Strategies of information processing. New York: Academic Press, 1978.

Greenwald, A. G. A double stimulation test of ideomotor theory with implications for selective attention. Journal of Experimental Psychology, 1970, 84, 392-398.

Herrmann, D. J., \& KAY, B. E. Familiarity and organization of category terms in semantic memory. Memory \& Cognition, $1977,5,139-145$.

KinSNer, K., \& Sмith, M. C. Modality effects in word identification. Memory \& Cognition, 1974, 2, 637-640.

LEWIS, J. L. Semantic processing with bisensory stimulation. Journal of Experimental Psychology, 1972, 96, 455-457.

MCNeill, D., \& Lindig, K. The perceptual reality of phonemes, syllables, words and sentences. Journal of Verbal Learning and Verbal Behavior, 1973, 12, 419-430.

MynatT, B. T. Reaction times in a bisensory task: Implications for attention and speech perception. Journal of Experimental Psychology: Human Perception and Performance, 1977, 3, 316-324.

Posner, M. I., \& Hanson, V. L. Analyzing spoken and written language. In R. A. Cole (Ed.), Perception and production of fluent speech. Hillsdale, N.J: Erlbaum, 1980.

Reıcher, G. M. Perceptual recognition as a function of meaningfulness of stimulus material. Journal of Experimental Psychology, 1969, 81, 274-280.

Rollins, H. A., \& Hendricks, R. Processing of words presented simultaneously to eye and ear. Journal of Experimental Psychology: Human Perception and Performance, 1980, 6, 99-109.

Rosch, E. Cognitive representation of semantic categories. Journal of Experimental Psychology: General, 1975, 104, 192-233.

Sen, A., \& Posner, M. I. The effect of unattended visual and auditory words on cross-modal naming. Bulletin of the Psychonomic Society, 1979, 13, 405-408.

Wheeler, D. D. Processes in word recognition. Cognitive Psychology, 1970, 1, 59-85.

\section{NOTES}

1. The term "levels," as used here, is not meant to imply serial processing. The term is used only to represent a logical hierarchy of abstraction.

2. Due to equipment failures, subjects were not always able to complete each block of an experimental session. The equipment failures were random, however, and thus did not systematically influence which blocks were not completed. 


\section{Appendix}

Stimulus Words Listed by Category

\begin{tabular}{|c|c|c|}
\hline \multirow[b]{2}{*}{ Category } & \multicolumn{2}{|c|}{ Stimulus Words } \\
\hline & Target Phonemes Present & Target Phonemes Absent \\
\hline $\begin{array}{l}\text { Furniture } \\
\text { Relatives } \\
\text { Parts of the Body } \\
\text { Clothing } \\
\text { Animals } \\
\text { Vegetables }\end{array}$ & $\begin{array}{l}\text { sofa, desk, dresser, bookcase, stool, stove } \\
\text { sister, cousin, niece, husband, son, parents } \\
\text { legs, arms, eyes, nose, ears, stomach } \\
\text { sock, pants, skirt, blouse, dress, sweater } \\
\text { horse, mouse, squirrel, fox, skunk, hamster } \\
\text { peas, lettuce, spinach, asparagus, celery, beets }\end{array}$ & $\begin{array}{l}\text { chair, table, bed, lamp, couch, mirror } \\
\text { aunt, uncle, brother, nephew, daughter, wife } \\
\text { head, foot, finger, hand, mouth, neck } \\
\text { shirt, shoe, coat, hat, tie, jacket } \\
\text { cat, cow, sheep, goat, wolf, camel } \\
\text { carrot, corn, potato, tomato, broccoli, cabbage }\end{array}$ \\
\hline
\end{tabular}

(Received for publication May 21, 1980;

revision accepted July 23, 1980.) 